\title{
CORRECTIONS
}

\section{Syria: tales of life, death, and dignity}

In this Personal View by Samer Jabbour (BMJ 2012;344:e1691, doi:10.1136/bmj.e1691) an editing error resulted in a mistake in the paragraph titled “December 1996." Yassin Al Haj Saleh's "trial" is noted as having taken place in 1982 when it did not, in fact, take place until 1992. 